\title{
Non-Protein-Bound Transition Metals and Hydroxyl Radical Generation in Cerebrospinal Fluid of Newborn Infants with Hypoxic Ischemic Encephalopathy
}

\author{
TOHRU OGIHARA, KAZUYA HIRANO, HIROMI OGIHARA, KIRYO MISAKI, MAYO HIROI, \\ TAKAO MORINOBU, HAN-SUK KIM, SATORU OGAWA, RYOICHI BAN, \\ MASASHI HASEGAWA, AND HIROSHI TAMAI \\ Department of Pediatrics, Division of Neonatology, Osaka Medical College, Osaka, Japan
}

\begin{abstract}
Among various hypothetical mechanisms for the in vivo production of reactive oxygen species, transition metalcatalyzed reactions in cooperation with a biologic reducing agent like ascorbic acid or superoxide may be some of the most important. In the present study, we retrospectively examined the existence of non-protein-bound metal ions, an essentially hazardous pro-oxidant form of various transition metals, and the occurrence of metal-catalyzed reactive oxygen species production in cerebrospinal fluid (CSF) of 10 infants with hypoxic ischemic encephalopathy (HIE) subsequent to perinatal asphyxia and 12 control infants within $72 \mathrm{~h}$ of birth. Non-protein-bound iron was detected in eight out of 10 CSF samples from the HIE infants and its level was significantly correlated with Sarnat's clinical stage, whereas none of the control infants had detectable non-protein-bound iron levels. Non-protein-bound copper was below the detection limit in all CSF samples from both groups. Ascorbic acid was significantly increased in the CSF of HIE infants when compared with that of controls (means, 664.9 versus $449.4 \mu \mathrm{M}, p=0.008$ ). ortho-Tyrosine and meta-tyrosine, which are highly specific and sensitive markers of protein oxidation induced by hydroxyl radicals, were significantly higher in HIE infants than in controls when evaluated by the ratio relative to their source amino acid, phenylalanine [means, 110.5 versus
\end{abstract}

\section{ABSTRACT}

75.4, $p=0.018$ for ortho-tyrosine/phenylalanine; 104.6 versus $67.7\left(\mathrm{nM} / \mu \mathrm{M} \times 10^{2}\right), p=0.048$ for $m e t a$-tyrosine/phenylalanine]. Both ratios were significantly correlated with non-proteinbound iron, but not with ascorbic acid. Our preliminary observations provide direct evidence that hydroxyl radicals are generated in the CNS during asphyxiation. Iron chelation therapy could be worth developing as a neuroprotective strategy for perinatal asphyxia. (Pediatr Res 53: 594-599, 2003)
Abbreviations
ROS, reactive oxygen species
HIE, hypoxic ischemic encephalopathy
CSF, cerebrospinal fluid
NPBI, non-protein-bound iron
NPBC, non-protein-bound copper
o-Tyr, ortho-tyrosine
m-Tyr, meta-tyrosine
Phe, phenylalanine
AA, ascorbic acid
DHAA, dehydroascorbic acid
UA, uric acid
GC/MS, gas chromatography-mass spectrometry

Advanced perinatal and obstetric management cannot yet prevent brain damage in newborn infants after perinatal asphyxia. Current therapeutic strategies for HIE occurring after perinatal asphyxia are not specific and are only supportive. Numerous studies have suggested that free radicals could have a key role in causing hypoxic ischemic damage to the brain, especially during the reoxygenation/reperfusion phase $(1,2)$.

Received February 22, 2002; accepted October 16, 2002.

Correspondence: Tohru Ogihara, M.D., Ph.D., Department of Pediatrics, Division of Neonatology, Osaka Medical College, 2-7, Daigaku-machi, Takatsuki, Osaka 569-8686, Japan; e-mail: ped025@poh.osaka-med.ac.jp

DOI: 10.1203/01.PDR.0000054685.87405.59
Among various hypothetical mechanisms for the production of ROS in living organisms, transition metal-catalyzed reactions may be some of the most important (3). When transition metals like iron or copper exist in their non-protein-bound "free" form in vivo, these metals can convert less reactive radicals to more reactive species. Thus, the human body is normally very careful to sequestrate these metal ions to be incorporated into specific metal-binding proteins. However, it is known that iron can be liberated from iron-storage ferritin when environmental $\mathrm{pH}$ decreases sufficiently, which may occur during perinatal asphyxia (4).

Several studies have detected NPBI in the plasma of newborn infants, who show immature iron metabolism (5-7). In 
infants with severe perinatal asphyxia, a close association has been reported between an adverse outcome and the plasma concentration of NPBI (8). In addition, CSF essentially has no significant binding capacity for iron or copper because its content of transferrin and ceruloplasmin is very low (9), and it was recently reported that NPBI is elevated in the CSF of preterm infants with posthemorrhagic ventricular dilatation (10). In this context, several animal studies have shown the effectiveness of iron chelation therapy for post hypoxia/ ischemia-reperfusion injury of the brain $(11,12)$.

In the brain, various nonradical compounds, such as catecholamines, ascorbic acid, thiols, and lipid peroxides, can produce reactive radical species in the presence of transition metal ions (9). Among them, AA is known to be strongly concentrated in the brain tissue and CSF by active transport mechanisms (13). In neonates with birth asphyxia, the AA concentration of CSF is known to increase markedly above the normal nonasphyxia level (14). AA, together with UA, is an integral water-soluble antioxidant that seems to serve as the main defense against oxidizing species in the aqueous phase of human body fluids. However, it has also been well confirmed that AA can contribute to the formation of highly reactive hydroxyl radicals in cooperation with redox active metal ions (15). Thus, we hypothesized that ROS formation catalyzed by redox-active transition metals may take place when HIE occurs after perinatal asphyxia.

In the present study, we measured the concentrations of NPBI, NPBC, and AA in the CSF of infants with HIE and matched controls, together with the oxidation products of AA and UA (DHAA and allantoin) as markers of ROS generation (16), $\mathrm{F}_{2 \alpha}$-isoprostane as a marker of lipid peroxidation (17), and the levels of $o$-Tyr and $m$-Tyr as highly specific and sensitive markers of protein oxidation induced by hydroxyl radicals $(18,19)$. We also analyzed whether these parameters were correlated with other well-established markers of HIE, including hypoxanthine (a marker of brain ischemia/hypoxia) (20), neuron-specific enolase (a marker of neuronal damage) (21), and excitatory amino acids (22).

\section{MATERIALS AND METHODS}

Patients. Among the infants admitted to the Neonatal Intensive Care Unit of Osaka Medical College Hospital between April 1999 and March 2001, term infants (gestational age $\geq 37$ wk) who had abnormal neurologic signs as described by Sarnat and Sarnat (23), such as increased irritability and jitteriness, abnormal tone, abnormal primitive reflexes, altered consciousness, or convulsions, within the first $24 \mathrm{~h}$ of life and underwent lumbar puncture within $72 \mathrm{~h}$ of birth to rule out meningitis or subarachnoid hemorrhage associated with at least three of the following criteria were enrolled in this study: 1) signs of fetal distress, defined as an abnormal cardiotocogram (late deceleration, absent variability, or bradycardia) with thick meconium staining of amniotic fluid; 2) an Apgar score $\leq 6$ at 5 min after birth; 3 ) a requirement for more than 3 min of positive pressure resuscitation immediately after birth; and 4) umbilical cord blood $\mathrm{pH}<7.00$ or a base deficit $>20 \mathrm{mM}$.
Neurologically normal term infants without a history of perinatal asphyxia (no signs of fetal distress and an Apgar score $\geq 9$ at $5 \mathrm{~min}$ after birth), in whom lumbar puncture was performed within the first $72 \mathrm{~h}$ of life to exclude meningitis as part of a septic workup for suspected systemic infection, were used as controls.

Exclusion criteria for both groups were as follows: 1) congenital heart disease; 2) multiple malformations; 3) documented chromosomal abnormality; 4) an increase of leukocytes and/or erythrocytes in CSF; 5) macroscopic hemolysis in CSF supernatant; and 6) positive bacterial cultures of CSF or blood. During the study period, $10 \mathrm{HIE}$ and 12 control infants fulfilled the above criteria.

The study protocol was approved by the ethics committee of our hospital and investigations were only performed after informed consent had been obtained from the parents of the subjects.

Methods. CSF samples were immediately centrifuged to remove cell components and the supernatant was stored in plastic metal-free containers under nitrogen gas at $-80^{\circ} \mathrm{C}$ until being thawed once for analysis.

NPBI was measured by the method described previously based on bleomycin-iron complex dependent degradation of DNA (24). This assay only detects "free" iron and not iron bound to specific iron binding and storage proteins such as transferrin, ferritin, and $\mathrm{Hb}$. NPBC was measured by a similar method, using phenanthroline as a chelator instead of bleomycin (25). For both methods, the detection limit was $0.2 \mu \mathrm{M}$ and values of $<0.2 \mu \mathrm{M}$ were expressed as zero.

$o$-Tyr and $m$-Tyr were quantified by isotope dilution negative-ion chemical ionization GC/MS as their $n$-propyl heptafluorobutyryl derivatives (26) using a Shimadzu QP-5050A GC/MS system (Shimadzu, Kyoto, Japan). Three excitatory amino acids (glycine, aspartate, and glutamate) and phenylalanine were measured by electron-impact ionization GC/MS as their tert-butyldimethylsilyl derivatives (27) using a Shimadzu QP-5000 ver. 2 GC/MS system. $\mathrm{F}_{2 \alpha}$-Isoprostane was measured with a competitive enzyme immunoassay kit (Cayman Chemical, Ann Arbor, MI, U.S.A.). For the GC/MS analyses and the isoprostane assay, precipitation of protein with two volumes of ethanol was done before treatment of the sample to investigate only the non-protein-bound free form of each substance. Addition of $\mathrm{FeSO}_{4}$ at the final concentration of $100 \mu \mathrm{M}$ to the CSF samples before analysis did not influence the results of either measurement (data not shown).

AA, UA, and their oxidation products (DHAA and allantoin) were measured by HPLC according to the method of our previous report $(16,28)$. Hypoxanthine was also determined by HPLC (29) and the concentration of neuron-specific enolase was measured using an immunoradiometric assay kit (Eiken Chemical, Tokyo, Japan).

Isoprostane could not be assayed in one of the HIE infants and four of the control infants because CSF samples were insufficient.

Statistical analysis. Comparison of characteristic variables between groups was performed using the Mann-Whitney $U$ test for continuous variables and Fisher's exact test for categorical data. Differences between the two groups were assessed by 
Mann-Whitney $U$ test. For correlation analysis, Spearman's correlation coefficients were calculated. A $p$ value $<0.05$ was considered to be significant. Data were analyzed using SPSS statistical software (SPSS Inc., Chicago, IL, U.S.A.).

\section{RESULTS}

Clinical characteristics were comparable between the HIE and control groups, with the exception of the Apgar scores. Although the age at lumbar puncture varied widely because this examination was only performed for clinical indications, there was no statistical difference between the two groups (Table 1).

According to Sarnat and Sarnat (23), HIE was classified as stage 1 in four infants, stage 2 in three infants, and stage 3 in three infants. The outcome was normal for stage 1 and 2 infants. In contrast, all stage 3 infants had severe motor and mental disabilities as well as multicystic encephalomalacia with severe cortical atrophy on brain MRI at the age of 3-6 mo.

As shown in Figure 1, NPBI was detected in eight out of 10 CSF samples from the HIE infants, but none of the control infants had detectable NPBI levels in their CSF. Although the number of subjects was small, the concentration of NPBI in the CSF was strongly correlated with the clinical stage $(r=0.857$, $p=0.010)$ of the HIE infants. NPBC was below the detection limit in all samples from both groups (Table 2).

As shown in Table 2, AA and UA levels were significantly increased in the HIE infants when compared with the controls. The oxidation products of AA and UA, i.e. DHAA and allantoin, respectively, and the ratio of DHAA to AA were also significantly higher in the HIE infants, but no such difference was noted for the allantoin/UA ratio. CSF hypoxanthine and neuron-specific enolase levels were significantly higher in the HIE infants than in the controls. Excitatory amino acids, such as glycine, aspartate, and glutamate, did not differ significantly between the two groups, although the mean CSF level of each amino acid was about 2-fold higher in the HIE infants when compared with that in the control infants.

Figure 2 shows excellent chromatograms and resolution of $o$-Tyr and $m$-Tyr peaks obtained by selected ion monitoring of an $n$-propyl heptafluorobutyryl derivative from a CSF sample. The HIE infants had significantly higher $o$-Tyr and $m$-Tyr levels in the CSF when compared with the control infants (mean $\pm \mathrm{SD}, 20.6 \pm 18.6$ versus $8.7 \pm 2.6 \mathrm{nM}, p=0.002$ for

Table 1. Clinical characteristics of study neonates

\begin{tabular}{lcc}
\hline & HIE $(n=10)$ & Control $(n=12)$ \\
\hline Gestational age (wk) & $39(37-41)$ & $39(37-41)$ \\
Birth weight (g) & $2669(2280-4262)$ & $2772(2120-3840)$ \\
Gender (M/F) & $7 / 3$ & $5 / 7$ \\
Cesarean section $(n)$ & $3 / 10$ & $4 / 12$ \\
Apgar score & & \\
at 1 min & $3(0-4)^{*}$ & $9(6-10)$ \\
at 5 min & $5(1-6)^{*}$ & $10(9-10)$ \\
Age at lumbar puncture (h) & $25(5-69)$ & $48(8-72)$ \\
\hline
\end{tabular}

Values are expressed as the median (range). Differences between groups were assessed by Mann-Whitney $U$ test or Fisher's exact test as appropriate.

$* p<0.05$ vs control infants.

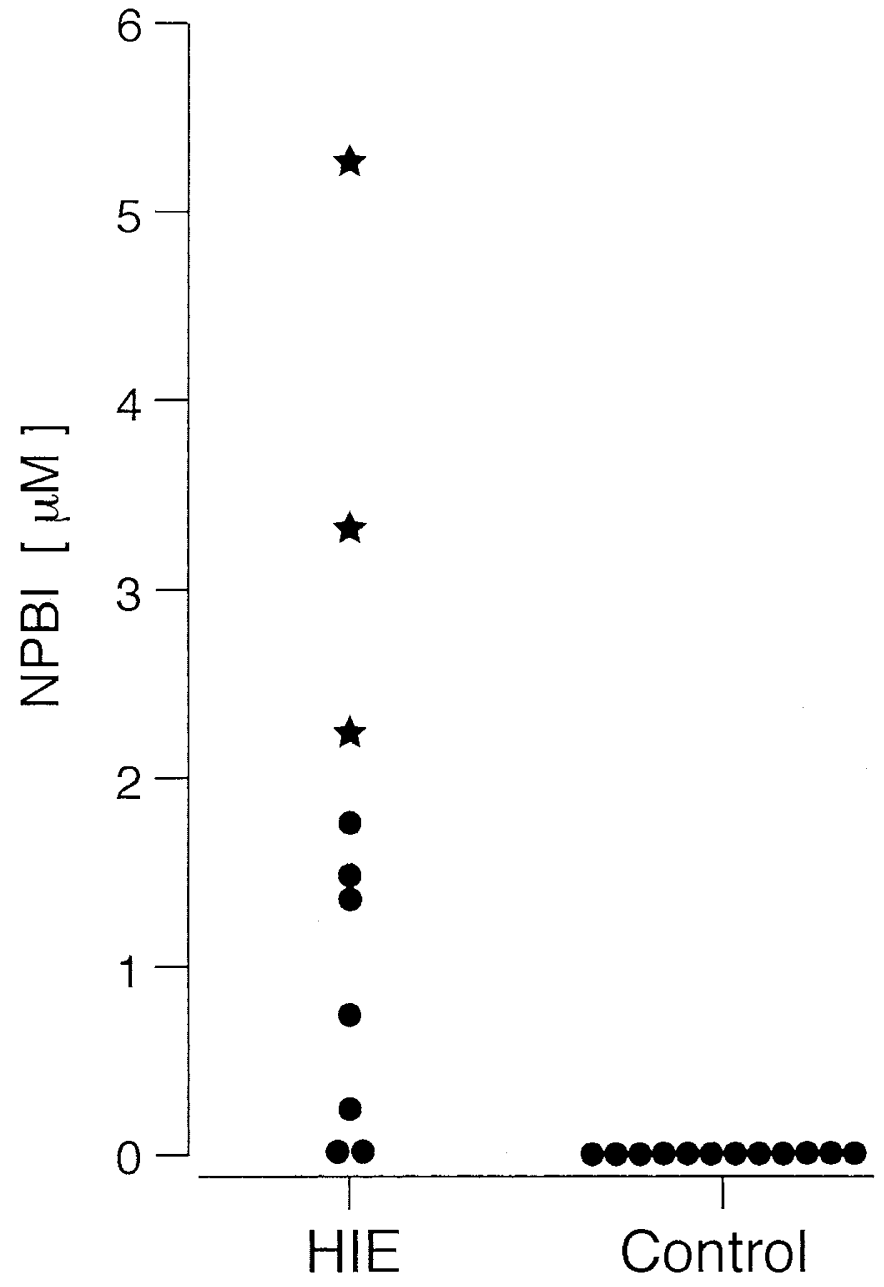

Figure 1. Concentration of NPBI in the CSF of newborn infants with HIE or control infants. Stars represent stage 3 HIE according to Sarnat and Sarnat (23).

Table 2. Non-protein-bound metal ions, major water-soluble antioxidants, and other biochemical parameters in the newborn infants with HIE and the control infants

\begin{tabular}{lccc}
\hline & & & $p$ \\
& HIE & Control & $\begin{array}{c}p \\
\text { Value }\end{array}$ \\
\hline NPBI & $1.6 \pm 1.6$ & $0.0 \pm 0.0$ & 0.002 \\
NPBC & $0.0 \pm 0.0$ & $0.0 \pm 0.0$ & - \\
AA & $664.9 \pm 213.6$ & $449.4 \pm 121.5$ & 0.008 \\
DHAA & $10.6 \pm 5.0$ & $4.0 \pm 4.2$ & 0.002 \\
DHAA/AA (\%) & $1.8 \pm 1.3$ & $0.9 \pm 1.0$ & 0.007 \\
UA & $136.5 \pm 176.1$ & $29.3 \pm 13.9$ & $<0.001$ \\
Allantoin & $2.2 \pm 0.8$ & $1.3 \pm 0.4$ & 0.014 \\
Alantoin/UA (\%) & $3.0 \pm 1.9$ & $5.1 \pm 2.5$ & 0.057 \\
Hypoxanthine & $11.7 \pm 10.7$ & $6.5 \pm 1.5$ & 0.032 \\
NSE (ng/mL) & $107.9 \pm 164.0$ & $16.7 \pm 4.1$ & 0.018 \\
Glycine & $19.4 \pm 17.7$ & $11.5 \pm 2.5$ & 0.129 \\
Aspartate & $1.9 \pm 2.6$ & $0.9 \pm 0.3$ & 0.391 \\
Glutamate & $5.1 \pm 4.3$ & $3.1 \pm 0.6$ & 0.510 \\
\hline
\end{tabular}

Values are expressed as the mean \pm SD. The unit of concentration was micromolar, unless otherwise stated in parentheses. Differences between groups were assessed by Mann-Whitney $U$ test. NSE, neuron-specific enolase.

$o$-Tyr and $20.5 \pm 24.9$ versus $8.0 \pm 3.1, p=0.021$ for $m$-Tyr) (Fig. $3 A$ ). The ratios of both tyrosine molecules to phenylalanine (the source amino acid) were also higher in the HIE 


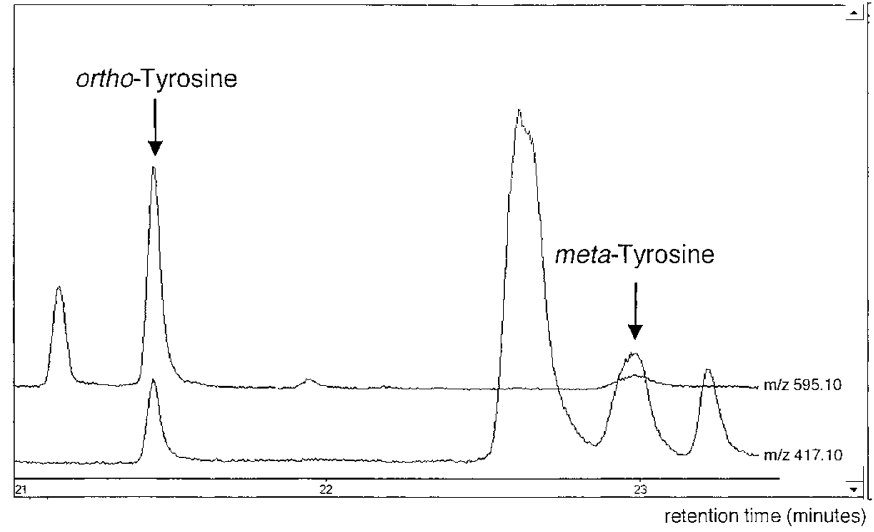

Figure 2. Typical selected-ion monitoring chromatograms of $o$-Tyr and $m$-Tyr at $\mathrm{m} / \mathrm{z} 417.10$ and 595.10 obtained from an $n$-propyl heptafluorobutyryl derivative of CSF by isotope dilution negative ion chemical ionization GC/MS analysis.

A
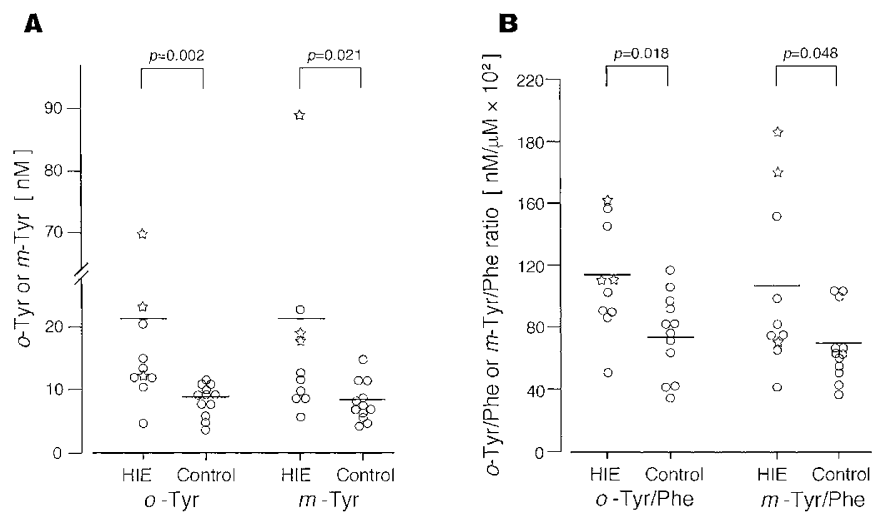

Figure 3. (A) $o$-Tyr and $m$-Tyr concentrations in the CSF of newborn infants with HIE or control infants. $(B)$ The ratios of these tyrosines to Phe. Stars represent stage 3 HIE according to Sarnat and Sarnat (23).

infants $\left[110.5 \pm 35.0\right.$ versus $75.4 \pm 25.5\left(\mathrm{nM} / \mu \mathrm{M} \times 10^{2}\right)$, $p=0.018$ for the $o-\mathrm{Tyr} / \mathrm{Phe}$ ratio and $104.6 \pm 55.0$ versus $67.7 \pm 23.5, p=0.048$ for the $m$-Tyr/Phe ratio] (Fig. $3 B$ ). In the HIE infants, the CSF levels of $o$-Tyr and $m$-Tyr were significantly correlated with the clinical stage $(r=0.642$, $p=0.048$ for $o$-Tyr and $r=0.779, p=0.019$ for $m$-Tyr), although the correlations of either ratio with stage were not statistically significant $(r=0.565, p=0.090$ for the $o$-Tyr/Phe ratio and $r=0.578, p=0.083$ for the $m$-Tyr/Phe ratio).

Correlations between the $o$-Tyr/Phe or $m$-Tyr/Phe ratio and the other biochemical parameters were assessed in 22 study subjects from both groups. These ratios showed a significant correlation with NPBI $(r=0.654, p=0.003$ for the $o$-Tyr $/$ Phe ratio and $r=0.507, p=0.020$ for the $m$-Tyr/Phe ratio), but not with the level of AA or the DHAA/AA ratio. Other parameters were also not significantly correlated with these ratios, except for hypoxanthine with the $o$-Tyr/Phe ratio $(r=0.500, p=$ $0.026)$ and neuron-specific enolase with the $o-\mathrm{Tyr} / \mathrm{Phe}$ ratio $(r=0.648, p=0.004)$.

The CSF concentration of $\mathrm{F}_{2 \alpha}$-isoprostane was significantly increased in the HIE infants when compared with the control infants $(177.3 \pm 67.1$ versus $65.9 \pm 28.0 \mathrm{pM}, p=0.001)$ (Fig.

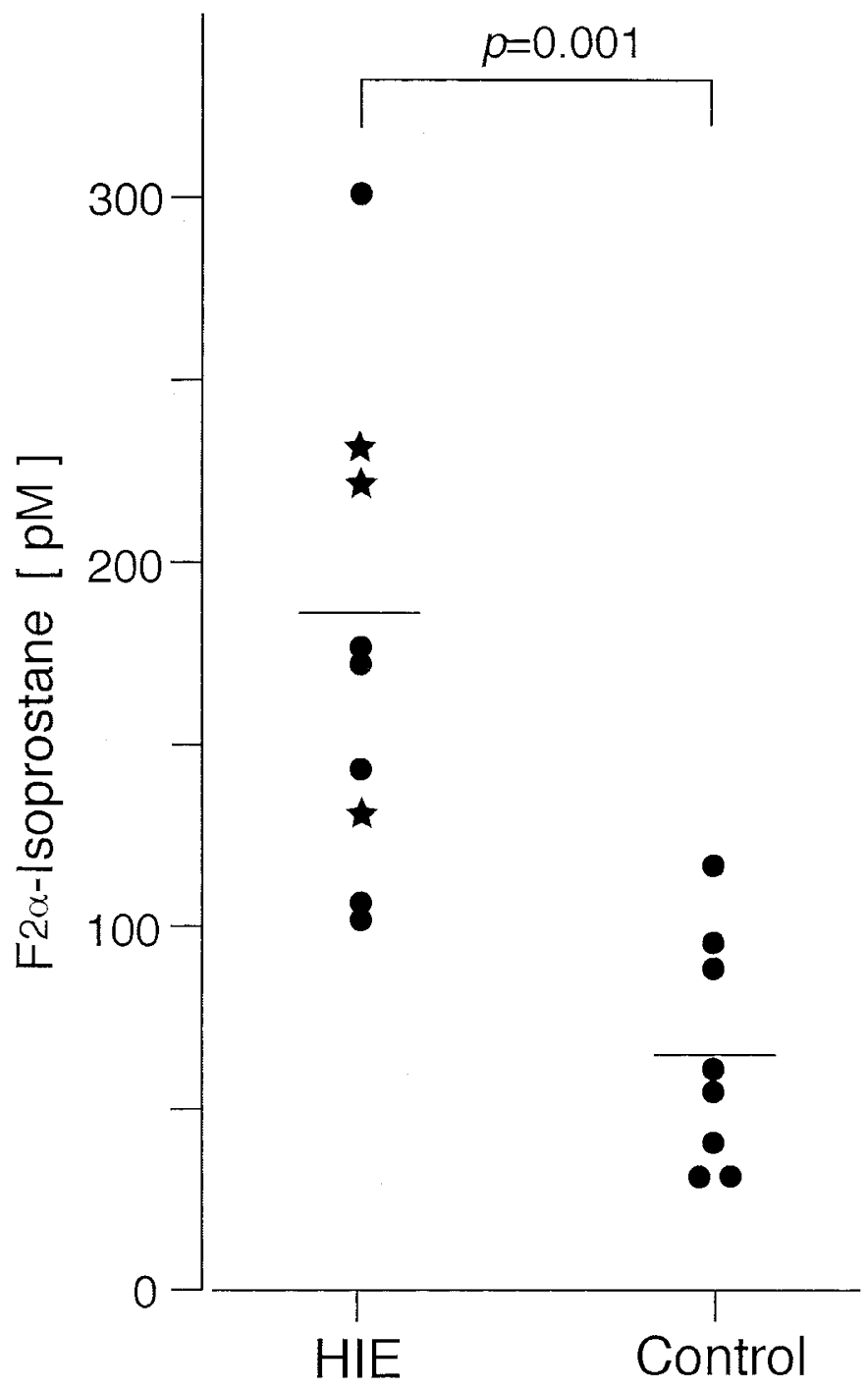

Figure 4. Concentration of $\mathrm{F}_{2 \alpha}$-isoprostane in the CSF of newborn infants with HIE or control infants. Stars represent stage 3 HIE according to Sarnat and Sarnat (23); horizontal bar $=$ mean.

$4)$, and it was significantly correlated with NPBI $(r=0.836$, $p=0.008)$, AA $(r=0.564, p=0.024)$, hypoxanthine $(r=$ $0.695, p=0.007), o-\mathrm{Tyr} / \mathrm{Phe}(r=0.598, p=0.017)$, or $m$-Tyr/Phe $(r=0.56, p=0.025)$ in all measurable samples, but was not significantly correlated with clinical stage in the HIE group $(r=0.58, p=0.1)$.

\section{DISCUSSION}

The most striking finding of the present study was the presence of NPBI in the CSF of HIE infants and not in any of the control infants. This is the first report of such a finding to the best of our knowledge. Newborn infants are essentially predisposed to releasing free iron due to their low iron-binding capacity because of the high iron saturation of transferrin and a decreased ferroxidase activity due to the low level of ceruloplasmin (7). Ferroxidase catalyzes the oxidation of ferrous iron to ferric iron and this process is essential for ionic forms of iron to be incorporated into transferrin. 
The CSF itself has no significant iron-binding capacity because its content of transferrin is very low and CSF transferrin is often close to iron saturation (9). The concentration of ceruloplasmin in CSF is also known to be quite low. In addition, the high AA level in HIE infants may possibly inhibit the ferroxidase activity of ceruloplasmin (30). Therefore, NPBI in CSF is likely to exist in the reduced ferrous form, which is thought to be an essentially pro-oxidant form of iron because of its ability to catalyze the formation of free radicals.

If the oxidized ferric form of NPBI comes into existence in CSF, it can be reduced to the ferrous state by some reductants with a lower redox potential than that of NPBI-Fe(III)/Fe(II) couple. Theoretically, the main candidates for such a role in $\mathrm{CSF}$ are glutathione (one-electron redox potential at $\mathrm{pH} 7.0$ of GSSG/GSSG ${ }^{\circ}=-1500 \mathrm{mV}$ versus a standard hydrogen electrode), superoxide $\left(\mathrm{O}_{2} / \mathrm{O}_{2}{ }^{-}=-330 \mathrm{mV}\right.$ ), and AA (DHAA/ ascorbate $\left.^{\cdot-}=-174 \mathrm{mV}\right)$, because the redox potential of low molecular mass iron, $\mathrm{Fe}(\mathrm{III}) / \mathrm{Fe}(\mathrm{II})$, is around $+100 \mathrm{mV}$ (31). Among them, it is unlikely that glutathione plays an important role because its concentration in CSF is very low (32). The higher DHAA/AA ratio in our HIE infants than in control infants suggested a possible role for AA in the reduction of ferric iron to ferrous iron in CSF, although it is also possible that the increased ratio may have resulted from the oxidation of AA acting as a sacrificial antioxidant to scavenge free radicals. In fact, it has been shown that, in the absence of ferroxidase activity, iron in the CSF remains in the ferrous state until AA is completely consumed during the incubation of CSF with ascorbate oxidase (33).

Non-protein-bound ferrous iron catalyzes the following reaction with hydrogen peroxide to yield hydroxyl radicals through Fenton chemistry (Eq. 1).

$$
\mathrm{Fe}^{2}+\mathrm{H}_{2} \mathrm{O}_{2} \rightarrow \mathrm{HO}^{\circ} \text { (hydroxyl radical) }+\mathrm{OH}^{-}+\mathrm{Fe}^{3+}
$$

Hydroxyl radicals can react with the aromatic ring of phenylalanine to generate the unnatural isomers $o$-Tyr and $m$-Tyr, instead of natural para- $(p-)$ tyrosine, after processing by phenylalanine hydroxylase (18). Because $o$-Tyr and $m$-Tyr are stable molecules and are not normally present in the body, they may serve as useful markers of oxidative protein damage induced by hydroxyl radicals (19). Although the precise and reliable quantification of these molecules is difficult because their concentrations in biologic fluids are usually extremely low, highly sensitive GC/MS analysis makes it possible. Significant elevation of $o$-Tyr and $m$-Tyr levels and their ratios to Phe in CSF from our HIE infants strongly supported the possible generation of hydroxyl radicals in the CNS during asphyxiation. As far as we are aware, this is also the first report of such an observation. Elevation of free iron and AA in CSF together with DHAA/AA ratio raises the possibility that hydroxyl radical production may take place via Fenton chemistry (Eq. 1) after NPBI reduction from the ferric to ferrous state by two-electron oxidation of AA. The good correlation of $o$-Tyr/ Phe and $m$-Tyr/Phe ratios with NPBI further supports this hypothesis. However, the lack of any association between these ratios and AA level or DHAA/AA ratio suggested that the absolute level of AA might not be directly related to hydroxyl radical generation. Although CSF concentration of AA was markedly raised in the HIE infants, being over four times that previously reported in adults (about $150 \mu \mathrm{M}$ ), the level in our control infants was also about three times the adult value. Therefore, if NPBI is not present, AA might not act as a pro-oxidant in CSF irrespective of its level. The possibility of hydroxylation of phenylalanine by peroxinitrite itself or peroxinitrite-derived hydroxyl radicals (34) could not be excluded because we did not estimate the contribution of nitric oxide by methods such as measurement of nitrotyrosine.

Once hydroxyl radicals have been formed, they attack biologic molecules (RH) at almost a diffusion-controlled rate and steal an electron, after which the radical chain reaction proceeds via the following sequence (Eq. 2-4) $(35,36)$.

$$
\begin{gathered}
\mathrm{RH}+\mathrm{HO}^{*} \rightarrow \mathrm{R}^{*}+\mathrm{H}_{2} \mathrm{O} \\
\mathrm{R}^{*}+\mathrm{O}_{2} \rightarrow \mathrm{ROO}^{\circ} \\
\mathrm{ROO}^{*}+\mathrm{RH} \rightarrow \mathrm{ROOH}+\mathrm{R}^{.}
\end{gathered}
$$

Ferrous iron also catalyzes the production of alkoxyl radical $\left(\mathrm{RO}^{\circ}\right)$ in the presence of an organic hydroperoxide $(\mathrm{ROOH})$ (Eq. 5), after which the radical chain reaction occurs (Eq. 6 and Eqs. 3 and 4) (36).

$$
\begin{gathered}
\mathrm{Fe}^{2+}+\mathrm{ROOH} \rightarrow \mathrm{RO}^{\cdot}+\mathrm{OH}^{-}+\mathrm{Fe}^{3+} \\
\mathrm{RO}^{\cdot}+\mathrm{RH} \rightarrow \mathrm{ROH}+\mathrm{R}^{.}
\end{gathered}
$$

Brain tissue is very rich in polyunsaturated fatty acids, which are especially sensitive to free radical attack (9). An increased CSF concentration of arachidonic acid metabolites has been reported in HIE infants (37). When RH is a polyunsaturated fatty acid, radical chain lipid peroxidation occurs, leading to the oxidative destruction of lipids. Significant elevation of isoprostane and its good correlation with NPBI support the occurrence of such processes.

Although the age of the two groups at lumbar puncture was quite different, the difference did not reach statistical significance ( $p=0.065$, Mann-Whitney $U$ test), and the variations in sampling time also showed no significant difference $(p=$ 0.088 , one-way ANOVA). In addition, since the study ended, four other control babies were studied within the first $24 \mathrm{~h}$ of life and the values of NPBI, AA, excitatory amino acids, and specific markers of oxidative damage did not differ significantly from the values for the control group (data not shown). Therefore, the differences in biochemical parameters between the asphyxiated infants and controls might not have originated from the difference in timing of the spinal tap.

Our results are strongly consistent with a previous report of a close association between an elevated plasma concentration of NPBI within $8 \mathrm{~h}$ after birth and an adverse outcome of severe birth asphyxia (8). Our findings also suggest that the marked effectiveness of iron chelators for ischemia-reperfusion injury in animal models $(11,12)$ or cultured cells may also be applicable for human birth asphyxia. Moreover, because NPBC was never detected in the CSF of HIE infants, it may be sufficient for only iron to be targeted as the metal for chelation. Although the clinical significance of our preliminary findings 
was not confirmed because of the small number of severely affected infants, we encourage the addition of iron chelation therapy to the list of neuroprotective strategies to be developed for perinatal asphyxia in the future.

Acknowledgments. The authors thank Drs. Hirohisa Tanaka, Shinya Oue, Seigo Hira, Akiko Akedo, Shigeo Yamaoka, Michi Ioka, Yuka Sano, and Kayoko Yamamoto for their kind assistance and advice; Hisakazu Takagi of Shimadzu Corp. for technical support; and Masumi Kobayashi for excellent laboratory assistance. We also thank Professor Etsuo Niki, chairman of Human Stress Signal Center, National Institute of Advanced Industrial Science and Technology for his valuable comments; and Etsuko Hata, Hitomi Takada, Satsuki Nakayama, and the nursing staff of the NICU for their help in sample collection.

This article is dedicated to Dr. Makoto Mino.

\section{REFERENCES}

1. Fellman V, Raivio KO 1997 Reperfusion injury as the mechanism of brain damage after perinatal asphyxia. Pediatr Res 41:599-606

2. Buonocore G, Perrone S, Bracci R 2001 Free radicals and brain damage in the newborn. Biol Neonate 79:180-189

3. Halliwell B, Gutteridge JMC 1990 Role of free radicals and catalytic metal ions in human disease: an overview. Methods Enzymol 186:1-85

4. Siesjo BK 1988 Acidosis and ischemic brain damage. Neurochem Pathol 9:31-88

5. Evans PJ, Evans R, Kovar IZ, Holton AF, Halliwell B 1992 Bleomycin-detectable iron in the plasma of premature and full-term neonates. FEBS Lett 303:210-212

6. Moison RMW, Palinckx JJS, Roest M, Houdkamp E, Berger HM 1993 Induction of lipid peroxidation of pulmonary surfactant by plasma of preterm babies. Lancet 341:79-82

7. Hirano K, Morinobu T, Kim HS, Hiroi M, Ban R, Ogawa S, Ogihara H, Tamai H, Ogihara T 2001 Blood transfusion increases radical promoting non-transferrin bound iron in preterm infants. Arch Dis Child Fetal Neonatal Ed 84:F188-F193

8. Dorrepaal CA, Berger HM, Benders MJNL, Van Zoeren-Grobben D, Van De Bor M, Van Bel F 1996 Non-protein-bound iron in postasphyxial reperfusion injury of the newborn. Pediatrics 98:883-889

9. Halliwell B 1992 Reactive oxygen species and the central nervous system. J Neurochem 59:1609-1623

10. Savman K, Nilsson UA, Blennow M, Kjellmer I, Whitelaw A 2001 Non-proteinbound iron is elevated in cerebrospinal fluid from preterm infants with posthemorrhagic ventricular dilatation. Pediatr Res 49:208-212

11. Palmer C, Roberts RL, Bero C 1994 Deferoxamine posttreatment reduces ischemic brain injury in neonatal rats. Stroke 25:1039-1045

12. Groenendaal F, Shadid M, McGowan JE, Mishra OP, Van Bel F 2000 Effects of deferoxamine, a chelator of free iron, on $\mathrm{Na}^{+}, \mathrm{K}^{+}$-ATPase activity of cortical brain cell membrane during early reperfusion after hypoxia-ischemia in newborn lambs. Pediatr Res 48:560-564

13. Grünewald RA 1993 Ascorbic acid in the brain. Brain Res Brain Res Rev 18:123-133
14. Oriot D, Bétrémieux P, Baumann N, Lefrançois C, Le Marec B 1992 CSF ascorbic acid and lactate levels after neonatal asphyxia: preliminary results. Acta Paediatr 81:845-846

15. Halliwell B 1996 Vitamin C: antioxidant or pro-oxidant in vivo? Free Radic Res 25:439-454

16. Ogihara T, Kim HS, Hirano K, Imanishi M, Ogihara H, Tamai H, Okamoto R, Mino M 1998 Oxidation products of uric acid and ascorbic acid in preterm infants with chronic lung disease. Biol Neonate 73:24-33

17. Morrow JD, Roberts LJ 1997 The isoprostanes: unique bioactive products of lipid peroxidation. Prog Lipid Res 36:1-21

18. Ishimitsu S, Fujimoto S, Ohta A 1984 Studies on the hydroxylation of phenylalanine by 6,7-dimethyl-5,6,7,8-tetrahydropteridine. Chem Pharm Bull (Tokyo) 32:752-756

19. Karam LM, Bergtold DS, Simic MG 1991 Biomarkers of OH radical damage in vivo. Free Radic Res Commun 12:11-16

20. Saugstad OD 1988 Hypoxanthine as an indicator of hypoxia: its role in health and disease through free radical production. Pediatr Res 23:143-150

21. Garcia-Alix A, Cabañas F, Pellicer A, Hernanz A, Stiris TA, Quero J 1994 Neuronspecific enolase and myelin basic protein: relationship of cerebrospinal fluid concentrations to the neurologic condition of asphyxiated full-term infants. Pediatrics 93:234-240

22. Roldán A, Figueras-Aloy J, Deulofeu R, Jiménez R 1999 Glycine and other neurotransmitter amino acids in cerebrospinal fluid in perinatal asphyxia and neonatal hypoxic-ischaemic encephalopathy. Acta Paediatr 88:1137-1141

23. Sarnat HB, Sarnat MS 1976 Neonatal encephalopathy following fetal distress: a clinical and electroencephalographic study. Arch Neurol 33:696-705

24. Gutteridge JMC, Rowley DA, Halliwell B 1981 Superoxide-dependent formation of hydroxyl radicals in the presence of iron salts: Detection of 'free' iron in biological systems by using bleomycin-dependent degradation of DNA. Biochem J 199:263-265

25. Gutteridge JMC 1984 Copper-phenanthroline-induced site-specific oxygen-radical damage to DNA. Detection of loosely bound trace copper in biological fluids. Biochem J 218:983-985

26. Leeuwenburgh C, Rasmussen JE, Hsu FF, Mueller DM, Pennathur S, Heinecke JW 1997 Mass spectrometric quantification of markers for protein oxidation by tyrosyl radical, copper, and hydroxyl radical in low density lipoprotein isolated from human atherosclerotic plaques. J Biol Chem 272:3520-3526

27. Mawhinney TP, Robinett RSR, Atalay A, Madson MA 1986 Analysis of amino acids as their tert-butyldimethylsilyl derivatives by gas-liquid chromatography and mass spectrometry. J Chromatogr 358:231-242

28. Ogihara T, Okamoto R, Kim HS, Nagai A, Morinobu T, Moji H, Kamegai M, Hirano K, Ogihara H, Tamai H, Mino M 1996 New evidence for the involvement of oxygen radicals in triggering neonatal chronic lung disease. Pediatr Res 39:117-119

29. Harkness RA 1988 Hypoxanthine, xanthine and uridine in body fluids, indicators of ATP depletion. J Chromatogr 429:255-278

30. Gutteridge JMC 1991 Plasma ascorbate levels and inhibition of the antioxidant activity of caeruloplasmin. Clin Sci 81:413-417

31. Buettner GR 1993 The pecking order of free radicals and antioxidants: lipid peroxidation, $\alpha$-tocopherol, and ascorbate. Arch Biochem Biophys 300:535-543

32. Janáky R, Ogita K, Pasqualotto BA, Bains JS, Oja SS, Yoneda Y, Shaw CA 1999 Glutathione and signal transduction in the mammalian CNS. J Neurochem 73:889902

33. Gutteridge JMC 1992 Ferrous ions detected in cerebrospinal fluid by using bleomycin and DNA damage. Clin Sci 82:315-320

34. Halliwell B, Zhao K, Whiteman M 1999 Nitric oxide and peroxynitrite. The ugly, the uglier and the not so good: a personal view of recent controversies. Free Radic Res 31:651-669

35. Gutteridge JMC 1992 Iron and oxygen radicals in brain. Ann Neurol 32:S16-S21

36. Minotti G, Aust SD 1992 Redox cycling of iron and lipid peroxidation. Lipids 27:219-226

37. Vilanova JM, Figueras-Aloy J, Rosello J, Gomez G, Gelpi E, Jimenez R 1998 Arachidonic acid metabolites in CSF in hypoxic-ischaemic encephalopathy of newborn infants. Acta Paediatr 87:588-592 